\title{
Rekonstruktion des Druckpotenzials für den unteren Grundwasserleiter im Münsterländer Kreidebecken im Falle eines ungehinderten Grubenwasseranstiegs im Grundgebirge
}

\author{
Maj-Britt Ottenjann ${ }^{1} \cdot$ Sandra Kons ${ }^{2} \cdot$ Johannes Meßer $^{2} \cdot$ Florian Werner $^{2} \cdot$ Michael Getta $^{3} \cdot$ Patricia Göbel $^{1}$ \\ Eingegangen: 27. September 2021 / Überarbeitet: 6. Dezember 2021 / Angenommen: 6. Dezember 2021 / Online publiziert: 21. Januar 2022 \\ (c) Der/die Autor(en) 2022
}

\section{Zusammenfassung}

Im Münsterländer Kreidebecken (NRW \& Niedersachsen, Deutschland) wird für das im gesamten Becken verbreitete untere Grundwasserleitersystem der Schichten des Cenomans und Turons (Untere Oberkreide) ein hydraulischer Druckausgleich zwischen dem Nord- und Südrand angenommen. Für den Fall eines ungehinderten Grubenwasseranstiegs im Grundgebirge (Karbon) würde sich in diesem Grundwasserleitersystem eine hydraulische Druckpotenzialfläche ausbilden (,,worstcase"-Szenario), welche sich ausgehend von hydrogeologisch begründbaren, hydraulischen Druckpunkten am Nord-, Ost und Südrand des Beckens über das Beckeninnere aufspannen lässt. Die auf den potenziellen hydraulischen Druckpunkten des Beckens basierende und mittels ArcGIS interpolierte Druckpotenzialfläche weist ein generelles Gefälle nach Westen mit ihrem höchstgelegenen Druckpunkt im Nordosten (Fosbergquelle bei Bielefeld) und ihrem niedrigsten Druckpunkt im Südwesten (Ruhrbogen in Oberhausen) auf. Die Druckpotenzialfläche ist zukünftig für die „,worst-case“-Betrachtung von Grubenwasseranstiegsszenarien in stillgelegten Bergwerken oder von aufsteigenden Tiefengrundwässern in oberflächennahe Grundwasserleitersysteme verwendbar.

Schlüsselwörter Grubenwasseranstieg $\cdot$ Hydraulische Druckpotenzialfläche $\cdot$ Worstcase-Szenario $\cdot$ Münsterland

Johannes Meßer

messer@ewlw.de

Michael Getta

getta.michael@eglv.de

2 Emscher Wassertechnik GmbH, Brunnenstr. 37, 45128 Essen,

Patricia Göbel

pgoebel@wwu.de Deutschland

1 Angewandte Geologie, WWU Münster, Corrensstr. 24, 48149 Münster, Deutschland

Emschergenossenschaft/Lippeverband, Grundwasserbewirtschaftung, Kronprinzenstr. 24, 45128 Essen, Deutschland 


\title{
Reconstruction of the pressure potential in the lower aquifer of the Münsterländer Kreidebecken in case of unhindered rising mine water in the basement
}

\begin{abstract}
The Münsterländer Kreidebecken (NRW \& Lower Saxony, Germany) is a cretaceous basin that contains a confined aquifer system in the layers of the cenomaniac and turon. This system is hydraulically influenced by the underlying basement (carboniferous) that was partly dewatered during decades of mining activity. In case of unhindered mine water rise ("worst-case" scenario) a piezometric surface is believed to re-establish that spans from the outer rim (unconfined) over the inside of the basin. On this rim points with hydrogeologically justifiable piezometric pressure were identified and used to interpolate a piezometric surface covering the whole basin. The interpolation was made using ArcGIS. It shows a surface that dips to the west with its highest elevation in the northeast (Fosberg spring near Bielefeld) and its lowest elevation in the southwest (Ruhrbogen in Oberhausen). The piezometric surface can be used in the future for "worst-case" analysis of mine water rise scenarios in closed mines and for the potential of deep groundwater rising through weak parts of the confining layer into shallow aquifer systems.
\end{abstract}

Keywords Mine water rise $\cdot$ Hydraulic pressure potential · "Worst-case" scenario · Münsterland

\section{Einleitung und Hydrogeologische Modellvorstellung des Münsterländer Kreidebeckens}

Das Münsterländer Kreidebecken stellt eine geschlossene Grundwasserlandschaft dar, welche durch ein dreischichtiges Grundwasserfließsystem gekennzeichnet ist (Michel 1995). Es besteht aus einem unteren Kluftgrundwasserleiter, einer fast wasserundurchlässigen Trennschicht und mehreren, nur lokal oberflächennah verbreiteten Porengrundwasserleitern (Michel 1995; siehe Abb. 1). Die Unterlage des Münsterländer Kreidebeckens, welche überwiegend aus Ablagerungen des Oberkarbons und lokal aus Gesteinen des Perms, der Trias, des Juras und der Unterkreide besteht, ist bis auf ein paar lokale Ausnahmen als schwer wasserdurchlässig einzustufen (Struckmeier 1990). Der südwestliche Rand des Münsterländer Kreidebeckens wurde durch den Einfluss der bergbaulichen Tätigkeiten, die die natürlichen Grundwasserverhältnisse der Region tiefgreifend verändert haben, geprägt (Michel 1995).

Der im überwiegenden Bereich an den Rändern des Münsterländer Kreidebeckens ausstreichende und zum Inneren des Beckens abtauchende untere Kluftgrundwasserleiter der Kreide (siehe Abb. 1) wird größtenteils aus Kalk- und Mergelsteinen der Oberkreide und untergeordnet in manchen Bereichen aus Sandsteinen der Unterkreide aufgebaut (Michel 1995). Er ist im Gegensatz zu den oberen lokalen Grundwasserleitern, regional im gesamten Münsterländer Kreidebecken verbreitet (Michel 1995). Im Westen des Beckens tritt der untere Grundwasserleiter zwischen Borken und Mülheim aufgrund der Überdeckung durch tonige tertiäre Schichten nicht zutage (Struckmeier 1990). Die erste untere, im gesamten Becken verbreitete Aquifereinheit ist der Grundwasserleiter des Cenomans (Struckmeier 1990). Dieser bildet im Großteil des Beckens gemeinsam mit den Schichten des Turons einen zusammenhängenden Aquiferkomplex (Struckmeier 1990). Im Norden und Osten des Münsterländer Kreidebeckens führen die tonig-mergelig ausgebildeten Labiatus-Schichten mit einer Mächtigkeit von $20-40 \mathrm{~m}$ zu einer Trennung dieses sonst zusammenhängenden Cenoman-Turon-Grundwasserleiters (Struckmeier 1990). Die Trennung in einen Cenoman-Grundwasserleiter und einen Turon-Grundwasserleiter ist als Quellenlinie sichtbar (Struckmeier 1990). Zum Beckeninneren nimmt die Wirkung dieser trennenden Schicht jedoch wieder ab (Struckmeier 1990).

Der im Hangenden folgende und mit einer Durchlässigkeit von nur $10^{-12}$ bis $10^{-9} \mathrm{~m} / \mathrm{s}$ nahezu undurchlässige $\mathrm{Em}$ scher-Mergel (Emscher-Formation) trennt mit einer Mächtigkeit von über $1500 \mathrm{~m}$ im Zentrum des Beckens den unteren Kluftgrundwasserleiter der Kreide von den oberflächennahen Porengrundwasserleitern, die dem Emscher-Mergel aufliegen (Michel 1995). In Richtung der Randbereiche des Beckens nimmt die Mächtigkeit des Emscher-Mergel sukzessive ab bis er gänzlich auskeilt. Hier treten die beiden Grundwasserleiter hydraulisch in Kontakt (Michel 1995).

Zudem kann der Emscher-Mergel in Tiefen von bis zu $50 \mathrm{~m}$ geklüftet sein und führt in diesen Bereichen Kluftgrundwasser (Coldewey und Wesche 2017). Im Grenzbereich zum überlagernden Quartär ist häufig eine tonigschluffige Verwitterungsschicht mit geringen Durchlässigkeitsbeiwerten ausgebildet, welche die beiden Grundwasserleiter voneinander trennt. Durch anthropogene Einflüsse und geologische Prozesse sind häufig tonige Verwitterungsschichten nicht durchgängig entwickelt, sodass hier angenommen werden kann, dass das Quartär und der geklüftete oberflächennahe Emscher-Mergel ein gemeinsames oberes Grundwasserstockwerk bilden.

In Regionen, wo die Mächtigkeit des Emscher-Mergels $50 \mathrm{~m}$ unterschreitet, kann ein hydraulischer Kontakt zwi- 


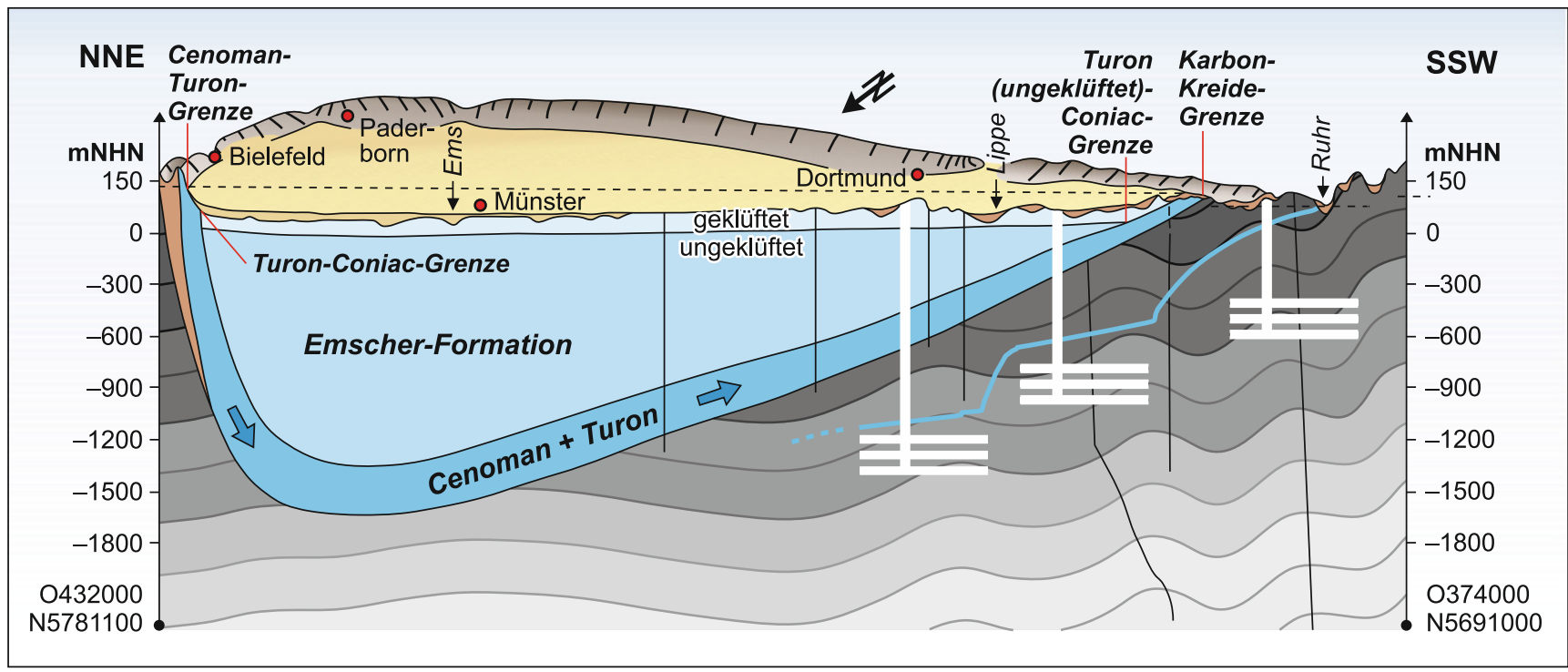

Abb. 1 Schematischer Schnitt durch das Münsterländer Kreidebecken. Blaue durchgezogene und gestrichelte Linie stellt den Grubenwasserspiegel im bergbaulich beeinflussten Südrand dar; schwarze nahezu horizontale Linie stellt die Druckpotenzialfläche im unteren Grundwasserleiter des Cenomans und Turons dar; tektonische Störungen werden durch schwarze senkrechte Linien dokumentiert. (@ Geowissenschaftliche Daten: Geologisches 3D-Landesmodell NRW 2018 @ Geologischer Dienst NRW 2021)

Fig. 1 Schematic section through the Münsterländer Kreidebecken. Blue solid and dashed line represents the mine water table in the mininginfluenced southern margin; black nearly horizontal line represents the hydraulic pressure potential in the lower aquifer of Cenomanian and Turonian; tectonic faults are documented by black vertical lines. (@ geoscientific data: Geologisches 3D-Landesmodell NRW 2018 @ Geologischer Dienst NRW 2021)

schen dem oberen und unteren Grundwasserstockwerk entstehen (Meßer 1997). Ebenso kann die sandige Ausbildung des Emscher-Mergels im Westen zum hydraulischen Kontakt der beiden Grundwasserstockwerke führen (Meßer 1997).

Für die Grundwasserströmung im unteren Grundwasserleiter der Kreide ist ein erhöhter Widerstand anzunehmen. Dies ist auf die Überdeckung durch den abdichtenden Emscher-Mergel und das tonig ausgebildete Tertiär im Westen des Beckens sowie auf die geringere Durchlässigkeit des Cenoman/Turonaquifers zum Beckeninneren hin, aufgrund der abnehmenden Klüftung und Verkarstung, zurückzuführen (Struckmeier 1990).

Im Ruhrgebiet führte die Grubenwasserförderung im $\mathrm{Zu}$ ge der bergbaulichen Tätigkeiten zu tiefgreifenden Veränderungen, sodass sich ein subregionales hydrologisches System ausgebildet hat, welches das regionale hydrologische System beeinflusst (Struckmeier 1990). Die Grubenwasserhaltung führte unter anderem zu einem Trockenfallen der Hellweg-Quellen (Meßer 1997) und der Abbau im Oberkarbon lokal zum Zerreißen der wasserstauenden Schichten des Cenomans und somit zu einer Verbindung der Grundwasserstockwerke der Oberkreide und des Steinkohlengebirges (Müller 1982). Für die Zeit des Nachbergbaus würde im Falle eines ungehinderten Grubenwasseranstiegs ein allgemeiner Druckanstieg in den Grundwasserleitern des Oberkarbons und den überlagernden Schichten der Oberkreide erfolgen. Dadurch könnten nahezu „natürliche“ vorberg- bauliche hydraulische Verhältnisse entstehen. Diese werden jedoch fortwährend durch hydraulische Wechselwirkungen von Strukturen, die im Zuge der bergbaulichen Aktivitäten entstanden sind, wie beispielsweise das unterirdische Netz der Erbstollen, beeinflusst. Wenn die Grundwasserdruckfläche im Grundwasserleiter des Oberkarbons die des unterer Grundwasserleiters übersteigt, könnte sich in Bereichen, wo Wasserwegsamkeiten im Gesteinsverband vorliegen und die aktuell einen Abstrom von oberflächennäherem Grundwasser in die tieferliegenden Grundwasserstockwerke aufweisen (Tiefenversickerungen), die Strömungsrichtung umkehren.

Bei der Betrachtung der Auswirkungen eines ungehinderten Grubenwasseranstiegs am südlichen Rand des Münsterländer Kreidebeckens sind die Wasserwegsamkeiten oder wasserstauenden Wirkungen der Oberkreideschichten für das aufsteigende tiefere Grundwasser und das abzuleitende hydraulische Druckpotenzial (Endzustand) von zentraler Bedeutung. Der überwiegend an den Beckenrändern ausstreichende untere Grundwasserleiter der Oberkreide stellt aufgrund seiner Verbreitung im gesamten Münsterländer Kreidebecken ein verbindendes Glied zwischen den Rändern des Beckens dar. Deshalb liegt der Fokus der Betrachtung der Auswirkungen eines ungehinderten Grubenwasseranstiegs auf dem großräumigen System des Münsterländer Kreidebeckens am Süd-, Nord- und Ostrand auf den Oberkreideschichten und ihren hydrogeologischen Eigenschaften. Im Südwesten treten die Oberkreideschichten 
aufgrund der überlagernden und als hydraulisch wirksame Trennschicht fungierenden Ablagerungen des Tertiärs (Meßer 1997) nicht zutage.

\section{Methodik}

Das Ziel der Arbeit ist die Herleitung einer hydraulischen Druckpotenzialfläche für den unteren regionalen Grundwasserleiter der Kreide im Münsterländer Kreidebecken im Falle eines ungehinderten Grubenwasseranstiegs (,,worstcase"-Szenario) im Grundgebirge (Karbon). Diese Arbeit basiert auf Ergebnissen des Forschungsprojektes „Auswirkungen eines ungehinderten Grubenwasseranstiegs sowie der Einstellung aller Poldermaßnahmen im Einzugsgebiet von Emscher und Lippe auf das oberflächennahe Grundwasser und die oberirdischen Gewässer" (Stifterverband „Forum Bergbau und Wasser“; Förderkennzeichen T0518/33420/2019). Die Darstellung des hydraulischen Druckpotenzials im nahezu gesamten Münsterländer Kreidebecken erfolgt in Form einer mittels ArcGIS interpolierten Fläche, die sich ausgehend von hydrogeologisch begründbaren hydraulischen Druckpunkten an den Rändern über nahezu das gesamte Becken aufspannen lässt. Bei den Druckpunkten handelt es sich um tiefliegende Austrittspunkte für Grundwasser, wie beispielsweise Quellen, Gewässer oder Erbstollen, die zu einer Druckentlastung des Grundwasserleiters führen können. Um eine Grundlage zur Ermittlung der relevanten hydraulischen Druckpunkte für die Ableitung des Druckpotenzials zu schaffen, erfolgte eine tiefergehende Betrachtung der (hydro-)geologischen Situation im gesamten Münsterländer Kreidebecken und in den für die Interpolation der Druckpotenzialfläche ausgewählten geologischen Blattgebieten an dessen Süd-, Nordund Ostrand.

Für die vorliegenden Arbeit wird angenommen, dass im unteren Grundwasserleiter der Kreide des Münsterländer Kreidebeckens, in dem die Durchlässigkeit mit der Tiefe abnimmt, die Grundwasserströmung geringer ist, aber dennoch weiterhin eine Weiterleitung des hydraulischen Drucks erfolgt. Des Weiteren wird angenommen, dass sich die ungehinderte Grundwasserströmung im unteren Grundwasserleiter der Kreide im regionalen Maßstab als horizontal-ebene Strömung vereinfacht und problemadäquat beschreiben lässt. Im Südwesten sowie am gesamten Westrand des Beckens wurden aufgrund mangelnder Ansatzpunkte keine weiteren Annahmen getroffen.

\section{Ergebnisse der Bestimmung der hydraulischen Druckpunkte}

\section{Südrand des Kreidebeckens}

Als Grundlage für die Herleitung der hydraulischen Druckpotenziale wurde zunächst eine hydrogeologische Modellvorstellung für den Südrand des Kreidebeckens erarbeitet. Betrachtet wurden dabei zunächst die Blattgebiete Mülheim, Essen, Bochum und Dortmund in einer tiefergehenden Literaturrecherche. Der Fokus lag dabei auf den Schichten des Karbons und der Oberkreide.

Im Allgemeinen liegt, wie aus den Erläuterungen und Schnitten zu den (ingenieur-)geologischen Karten (Bochum (Geologisches Landesamt Nordrhein-Westfalen 1988), Essen (Geologisches Landesamt Nordrhein-Westfalen 1992), Dortmund (Geologisches Landesamt Nordrhein-Westfalen 1987), Mülheim (Geologisches Landesamt NordrheinWestfalen 1986)) zu entnehmen ist, ein ähnlicher Schichtenaufbau in den Blattgebieten vor. Die Ablagerungen der Oberkreide beginnen im Liegenden mit dem Essener Grünsand des Cenomans. Der Essener Grünsand kann durch die bergbaulichen Tätigkeiten in seiner wasserstauenden Wirkung stellenweise beeinträchtigt sein (Geologisches Landesamt Nordrhein-Westfalen 1988). Müller (1982) beschreibt den Essener Grünsand im zentralen mittleren Revier als Wasserstauer mit großer Bedeutung, der jedoch im östlichen Ruhrrevier sehr fest ist, Kluftgrundwasser führt und im Westen als lockerer und porenwasserführender Sand vorliegt. Somit variiert die hydrogeologische Bewertung des Essener Grünsandes entlang des Südrandes des Münsterländer Kreidebeckens.

Anhand der gesammelten (hydro-)geologischen Informationen im Ruhrgebiet erfolgte die Auswahl einer Schichtgrenze, anhand derer Punkte ermittelt wurden, die der Ableitung von hydraulischen Druckhöhen am Südrand des Münsterländer Kreidebeckens im Hinblick auf einen ungehinderten Grubenwasseranstieg dienen. Es wurde die Annahme getroffen, dass das aufsteigende Grubenwasser im Karbon unter den überwiegend als Grundwassernichtleiter fungierenden Essener Grünsand (Oberkreide) drückt und an der Grenze vom Karbon zur überlagernden Kreide über tiefliegende Entlastungspunkte (im Folgenden als „Tiefpunkte“ bezeichnet), wie beispielsweise Gewässerläufe oder Talstrukturen, deren Verlauf die Karbon/ Kreidegrenze nahezu lotrecht schneidet, eine Entwässerung und damit Druckentlastung erfolgt. Die allgemeine Orientierung für die Auswahl der Tiefpunkte am Südrand des Münsterländer Kreidebeckens erfolgte daher in den Blattgebieten, in denen die Grenze zwischen der Kreide und dem Karbon verläuft. Zudem entspricht die Wahl der Karbon-Kreide-Grenze als Ausgangspunkt zur Herleitung der Druckhöhen aufgrund ihrer Höhenlage im Vergleich zu 


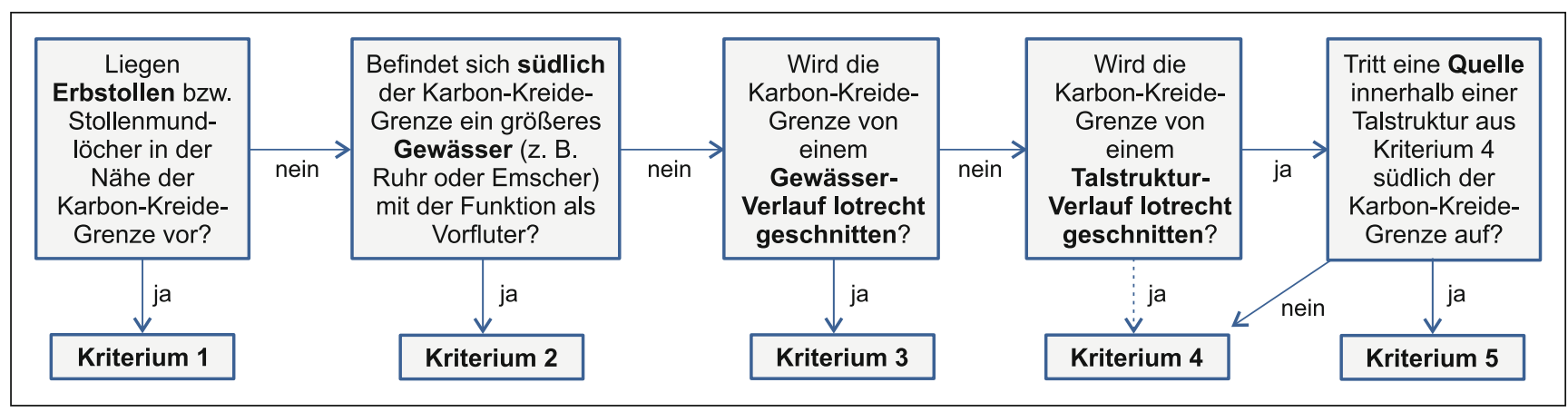

Abb. 2 Darstellung und Vorgehensweise bei der Wahl der Kriterien zur Festlegung der Tiefpunkte am Südrand des Münsterländer Kreidebeckens Fig. 2 Presentation and procedure of choosing the criteria for determining the low points at the southern boundary of the Münsterländer Kreidebecken

den zum Beckeninneren hin liegenden Schichtgrenzen, wie beispielsweise dem Übergang zum undurchlässigen Emscher-Mergel, am ehesten der Betrachtung eines ,worstcase"-Szenarios.

Im nächsten Schritt wurden Kriterien entwickelt, anhand derer die Auswahl von Tiefpunkten entlang der KarbonKreide-Grenze erfolgte. Die einzelnen Kriterien und deren Reihenfolge, nach welcher bei der Auswahl der Tiefpunkte vorgegangen wurde, sind in Abb. 2 aufgeführt. Diese Reihenfolge der Kriterien stellt eine generelle Priorisierung dar, welche jedoch im Zuge der Bearbeitung mancherorts, aufgrund des Auftretens besonderer örtlicher Gegebenheiten, nicht durchgehend beibehalten wurde.

Zunächst wurde das Kriterium 1 „Erbstollen“ bearbeitet. Für die im Zuge des Bergbaus zur Entwässerung der Gruben angelegten Erbstollen wurde die Annahme getroffen, dass diese auch in der Nachbergbauzeit als hydraulisch wirksame Elemente fungieren. In der Region liegen nach Tiedt (2012) Daten zu insgesamt 493 Stollenmundlöchern, die das Vorhandensein von Entwässerungsstollen kennzeichnen, vor. Die Stollenmundlöcher stellen den am tiefsten gelegenen Punkt des Erbstollens dar und dienen somit als Senke zur Entwässerung. Zunächst wurden die Stollenmundlöcher markiert, welche in der Nähe der Karbon-Kreide-Grenze liegen, bzw. die Stollenmundlöcher, deren anschließende Stollenverläufe zur Grenze verlaufen und dort zur Entwässerung der Karbon-Schichten führen.

Das Kriterium 2 greift in Gebieten, in denen südlich nahe der Karbon-Kreide-Grenze ein größeres Gewässer, wie beispielsweise die Ruhr oder Emscher, als Vorfluter fungiert. Ihre tiefere Lage im Gegensatz zu der Karbon-KreideGrenze ermöglicht die Entwässerung des Karbons in diesen Bereichen.

Kriterium 3 bezieht sich auf Schnittpunkte von Gewässern, deren Verlauf die Karbon-Kreide-Grenze lotrecht schneidet. An diesen Schnittpunkten würde das aufsteigende, unter die Kreide drückende Grubenwasser im Karbon im ,worst-case“-Szenario aufgrund der Entwässerung über diesen Tiefpunkt eine Druckentlastung erfahren. Die Schnittpunkte der Talstrukturen, deren Verläufe (Kriterium 4) die Karbon-Kreide-Grenze lotrecht schneiden, bauen auf der gleichen Annahme, welche auch dem Kriterium 3 zugrunde liegt, auf. Auch hier bilden die Schnittpunkte Tiefpunkte, welche als Druckentlastungspunkte durch die Entwässerung über diese sich in das Karbon und die Kreide einkerbenden Talstrukturen fungieren.

Falls Quellen aus dem Quellkataster NRW (Geologischer Dienst Nordrhein-Westfalen 2019b) in diesen Talstrukturen aus Kriterium 4 südlich der Karbon-Kreide-Grenze auftreten, greift das Kriterium 5 und die Quellpunkte werden anstatt des Schnittpunktes der Talstruktur und der Grenze als Tiefpunkte gewählt. An den Quellen würde dann bereits eine Druckentlastung des Karbons im Falle eines ungehinderten Grubenwasseranstiegs erfolgen.

Eine Auswahl an Tiefpunkten am Südrand des Münsterländer Kreidebeckens erfolgte für die Blattgebiete Duisburg, Mülheim an der Ruhr, Essen, Bochum, Witten, Schwerte und Kamen. In Bereichen, in denen mehrere potenzielle Tiefpunkte zur Auswahl standen, wurde dem tiefst gelegenen die höchste Priorität zugewiesen, da dieser bei einem ungehinderten Anstieg des Grubenwassers als erstes zu einer Druckentlastung führen würde. Bei der Auswahl der Tiefpunkte wurde zudem darauf geachtet, eine Überschätzung sowie eine Unterschätzung im Bezug auf das ,worst-case“-Szenario zu vermeiden. Des Weiteren wurde eine gleichmäßige Verteilung der Tiefpunkte am Südrand im Hinblick auf die Interpolation der Fläche mittels ArcGIS auf Grundlage der Tiefpunkte angestrebt. Für die potenziellen Tiefpunkte wurden die jeweiligen Höhen in ArcGIS ermittelt. Dies erfolgte über einen Verschnitt der Tiefpunkte mit dem DGM1 (Landesvermessung NRW 2020).

Die Vorgehensweise bei der Auswahl von Tiefpunkten in den Blattgebieten wird beispielhaft für das Blattgebiet Essen erläutert. Die Tiefpunkte wurden für jedes Blattgebiet nummeriert (z. B. E1 = Essen 1) und die Art des Kriteriums 


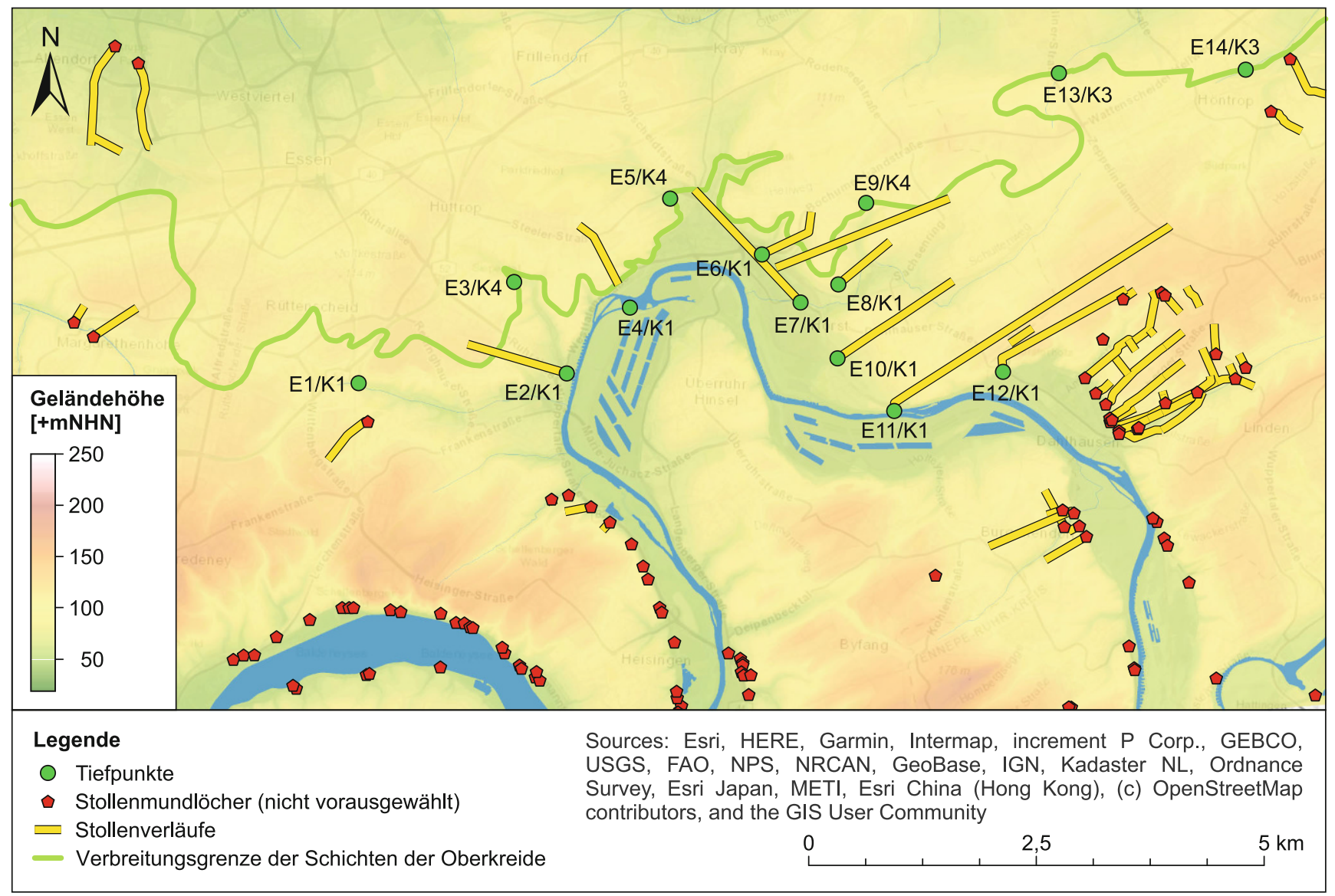

Abb. 3 Darstellung der potenziellen Tiefpunkte im Blattgebiet 4508 Essen (unter Verwendung der Daten von esri (2020); Geowissenschaftliche Daten: Geologisches 3D-Landesmodell NRW 2012 (Geologischer Dienst NRW 2019); Landesvermessung NRW (2020); LANUV (2020); Tiedt $(2012,2020))$

Fig. 3 Representation of the potential low points in the geological sheet area 4508 Essen (using data from esri (2020); geoscientific data: Geologisches 3D-Landesmodell NRW 2012 (Geologischer Dienst NRW 2019); Landesvermessung NRW (2020); LANUV (2020); Tiedt (2012, 2020))

(z. B. K1 (Kriterium 1)= Erbstollen/Stollenmundloch), dem der jeweilige Punkt angehört, gekennzeichnet.

Das Blattgebiet wird durch die Ruhr und das Vorkommen einer Vielzahl von Erbstollen dominiert (Abb. 3). Der Ruhrbogen verläuft inmitten des Blattgebietes nahe der KarbonKreide-Grenze (Kriterium 2). Die Ruhr fungiert hier im Falle eines ungehinderten Grubenwasseranstiegs als druckentlastendes Element. Ebenso sind die Erbstollen als hydraulisch wirksame Elemente zu nennen, deren Stollenmundlöcher sich in der Nähe der Ruhr oder ihrer Nebengewässer befinden (Kriterium 1). Des Weiteren treten in diesem Blattgebiet Talstrukturen auf, welche von der Ruhr nach Norden ziehen und dabei die Karbon-Kreide-Grenze schneiden (Kriterium 4). Die Talstrukturen liegen höher als die sich jeweils in ihrer Nähe befindlichen Stollenmundlöcher. Eine Druckentlastung des Karbons würde somit zunächst über die Erbstollen bzw. die Ruhr erfolgen. Da die Stollenmundlöcher auf dem Niveau der Ruhr liegen bzw. in diese entwässern, wurde die Ruhr (Kriterium 2) vernachlässigt. Die großen Ansammlungen der Erbstollen im Westen und Os- ten entlang der Ruhr und im Osten des Blattgebiets wurden ebenfalls vernachlässigt, da diese weit südlich der KarbonKreide-Grenze liegen. In die engere Auswahl fielen die Erbstollen, die die Karbon-Kreide-Grenze schneiden und deren Stollenmundlöcher direkt an der Ruhr bzw. an einem ihrer Nebengewässer liegen. Die finale Wahl im Blattgebiet Essen fiel auf die Stollenmundlöcher „Kunstwerk“ (E2/K1) und „Mecklingsbänker Erbstollen“ (E7/K1).

Analog zu der Vorgehensweise im Blattgebiet Essen erfolgte die Auswahl von Tiefpunkten in den Blattgebieten Mülheim, Bochum, Witten, Schwerte und Kamen.

Die Auswahl des Tiefpunktes im Blattgebiet Duisburg erfolgte im Anschluss an die Bearbeitung der anderen Blattgebiete. Um die Fläche weitestgehend nach Westen zu erweitern, wurde ein zusätzlicher Tiefpunkt im Blattgebiet Duisburg ausgewählt. In dieser Region sind die als hydraulisch wirksame Trennschicht fungierenden Schichten des Tertiärs verbreitet (Meßer 1997). Im Südwesten des Münsterländer Kreidebeckens ist der Emscher-Mergel zudem aufgrund seines Sandgehalts durchlässig (Struckmeier 
1990). Es besteht die Annahme, dass eine Druckentlastung des Systems in diesem Blattgebiet nicht in der Verbreitung des Tertiärs zu erwarten ist. Dementsprechend wurde der Punkt (S1) im Ruhrbogen in Oberhausen-Alstaden östlich der Kreide-Tertiär-Grenze in der Verbreitung des sandigen Emscher-Mergels ausgewählt.

Es wurden von 83 vorausgewählten Tiefpunkten insgesamt elf Tiefpunkte am Südrand final ausgewählt und von $\mathrm{S} 1$ bis S11 durchnummeriert (siehe Abb. 5 und Tab. 1 im Anhang). Die Höhenniveaus der Tiefpunkte steigen in einer West-Ost-Betrachtung von $+25 \mathrm{mNHN}$ auf $+100 \mathrm{mNHN}$ an.

\section{Nordrand des Kreidebeckens}

Als Grundlage zur Auswahl von hydraulischen Druckpunkten am Nordrand des Münsterländer Kreidebeckens erfolgte zunächst die Auswahl einer (hydro-)geologischen Grenze, die als Pendant zur Karbon-Kreide-Grenze am Südrand fungiert. Der untere regionale Grundwasserleiter verbindet den Nord- und Südrand hydraulisch miteinander. Daher liegt der Fokus in der (hydro-)geologischen Betrachtung der Blattgebiete am Nordrand auf den Schichten der Oberkreide. Im Zuge der Literaturrecherche wurden zunächst die Erläuterungen zu den Geologischen Karten der am Nordrand im Bereich des Ausstrichs der Oberkreideschichten gelegenen Blattgebiete (Ibbenbüren (Tecklenburg), Hasbergen, Lengerich, Bad Iburg (Iburg), Dissen (Borgholzhausen), Bockhorst, Halle (Westf.), Bielefeld, Brackwede) herangezogen. Die Datenlage variiert zwischen den Blattgebieten, da für manche Blätter keine neuere Auflage der Geologischen Karte 1:25.000 (GK 25) und der zugehörigen Erläuterung durch das Geologische Landesamt vorliegt, sondern ausschließlich Informationen aus den Erläuterungen zur Preußischen Geologischen Karte 1:25.000 existieren. Anhand von Informationen aus ELWAS-Web und den Erläuterungen zur Hydrogeologischen Karte 1:100.000 von Gütersloh wurden die Informationen am Nordrand ergänzt.

In der betrachteten Region bieten sich zwei Schichtgrenzen im Bereich der Oberkreide an, die durch eine Überlagerung von höher durchlässigeren durch gering durchlässige Ablagerungen gekennzeichnet sind und daher markante hydrogeologische Grenzen darstellen.

Als Beispiel wird im Folgenden die Situation im Blattgebiet Tecklenburg dargestellt. Laut Hydrogeologischer Karte (Blatt Ibbenbüren, Geologischer Dienst 2001) weist der auf die Unterkreide folgende Cenoman-Mergel eine sehr geringe Durchlässigkeit (Durchlässigkeitsklasse VI) auf. Die darauffolgenden Varians-Pläner, sowie die Labiatus- und Lamarcki-Schichten des Turons werden als mäßig bis mittel durchlässig (IV-III) eingestuft. Die sich jeweils im Hangenden befindlichen Schichten des Cenomans (CenomanKalk) und Turons (Scaphiten- und Schloenbachi-Schichten) werden als Kluftgrundwasserleiter mit einer mittleren bis hohen Durchlässigkeit (III-II) bezeichnet. Auf die Ablagerungen des Turons folgt der Emscher-Mergel des Coniac und Santon, dessen Unterkante als wichtige hydrologische Grenze angegeben wird. Eine weitere wichtige hydrogeologische Grenze befindet sich gemäß Geologischem Landesamt Nordrhein-Westfalen (1970) an der Oberkante des Rhotomagense-Kalkes (Grenze Cenoman-Turon).

Die Schichtgrenzen Cenoman-Turon und Turon-Coniac sind durch das Auftreten von Quellen gekennzeichnet, über die das System entwässert wird. Diese Quellen können somit als Basis zur Ableitung von Druckhöhen dienen. Beide Grenzen am Nordrand stellen Druckhöhen im unteren regionalen Cenoman-Turon-Grundwasserleiter dar, welcher den Nord- und Südrand hydraulisch miteinander verbindet. Die Cenoman-Turon-Grenze liegt im Vergleich zu der weiter im Beckeninneren liegenden Turon-Coniac-Grenze topographisch höher. Die Überdeckung der Turon-ConiacGrenze durch gering bis mäßig durchlässige quartäre Ablagerungen erschwert die genaue Schicht-Zuordnung der dort auftretenden Quellen und damit das Abgreifen des hydraulischen Druckpotenzials. Im Zuge der Betrachtung eines „worst-case“-Szenario fiel die Wahl auf die höchstgelegene ableitbare Druckhöhe, welche in diesem Falle die Quellenlinie an der Cenoman-Turon-Grenze darstellt.

Für das Blattgebiet Tecklenburg wurde der Höhenunterschied zwischen den zwei zur Auswahl stehenden Grenzen exemplarisch für den Bereich der Quelle N2 „Am Klee“ ermittelt. Die Grenzen liegen in diesem Bereich in ca. $900 \mathrm{~m}$ Entfernung voneinander. Die Cenoman-Turon-Grenze befindet sich hier auf einer Höhe von ca. $+75 \mathrm{mNHN}$. Die weiter zum Beckeninneren gelegene Turon-Coniac-Grenze liegt auf einer Höhe von ca. $+51 \mathrm{mNHN}$. Die Höhendifferenz der beiden Grenzen beträgt somit ca. $24 \mathrm{~m}$. Zum Vergleich wurden für zwei weitere Bereiche im Blattgebiet die Höhenunterschiede zwischen den beiden Grenzen bestimmt. Diese liegen bei $17 \mathrm{~m}$ und $28 \mathrm{~m}$.

Die Cenoman-Turon-Grenze lässt sich über die gesamten Blattgebiete verfolgen und bietet daher eine gute Grundlage zur Ableitung der dazugehörigen Quellen. Die CenomanTuron-Grenze (= Cenoman-Kalk/labiatus-Schichten-Grenze) stellt ein nachvollziehbares Pendant zu der am Südrand gewählten Karbon-Kreide-Grenze dar und bildet somit eine gute Grundlage zur Ableitung des hydraulischen Druckpotenzials am Nordrand.

Um eine Auswahl an Quellen entlang dieser Grenze treffen zu können, wurden alle Quellpunkte des Quellkatasters NRW, welche sich direkt an dieser Grenze befinden, markiert. Ebenso wurden Quellen ausgewählt, die südlich nahe der Grenze liegen, da das an der Grenze des Cenoman-Kalk zu den undurchlässigeren Labiatus-Schichten aufsteigende Grundwasser in die im Hangenden folgenden quartären $\mathrm{Ab}$ lagerungen eintritt und in diesen dem Gefälle des Beckens nach Richtung Süden fließt. Somit kann sich der Austritts- 


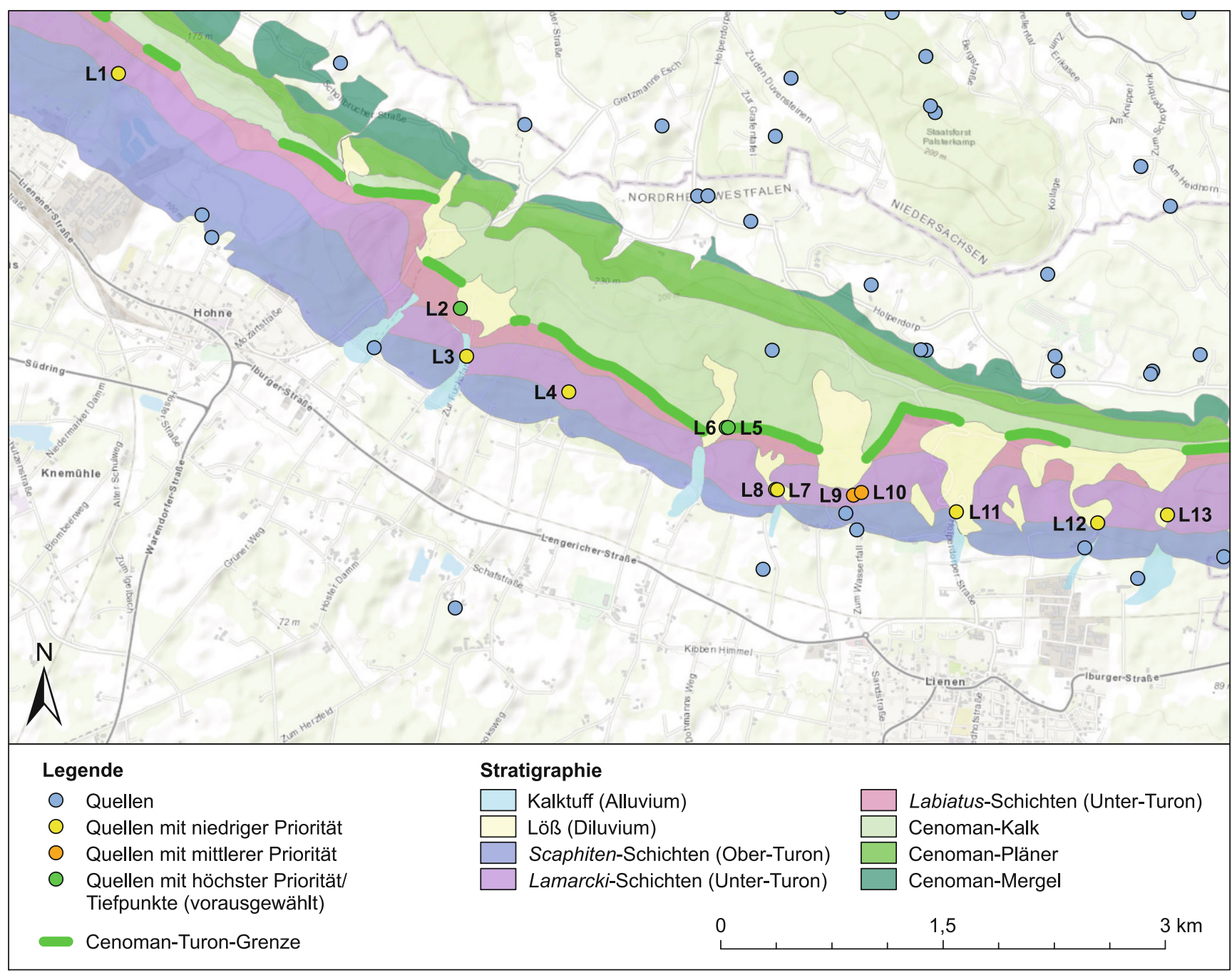

Abb. 4 Darstellung der Lage und Priorisierung der Quellen im Blattgebiet 3813 Lengerich (unter Verwendung Geowissenschaftlicher Daten: Geologisches 3D-Landesmodell NRW 2012 (Geologischer Dienst NRW 2019); Preußische geologische Landesanstalt (1935))

Fig. 4 Representation of the location and prioritization of the springs in the geological sheet area 3813 Lengerich (using geoscientific data: Geologisches 3D-Landesmodell NRW 2012 (Geologischer Dienst NRW 2019); Preußische geologische Landesanstalt (1935))

punkt der ausgewählten Quellenlinie zum Beckeninneren hin verschieben.

Nach Auswahl aller in Frage kommender Quellen wurde an diesen eine Priorisierung vorgenommen, um die Wahl auf eine geringere Anzahl an Quellen, die sich in gewissen Abständen über den Nordrand verteilen, auszudünnen. Die Anzahl der Punkte am Nordrand wurde somit der am Südrand angepasst, um eine ausgeglichene Ausgangslage für die Interpolation der hydraulischen Druckfläche zu schaffen. In die Priorisierung der Quellen floss in erster Linie die Distanz zur Cenoman-Turon-Grenze ein, sodass Quellen, die näher an der Grenze liegen, eine höhere Priorität erhielten. Die Quellen wurden je nach Distanz zur Grenze in drei Prioritätsstufen eingeteilt. Je weiter die Quellen von der Cenoman-Turon-Grenze entfernt liegen, desto geringer ist die Wahrscheinlichkeit, dass diese dem Cenoman-
Kalk zugeordnet werden können, auch wenn sie in Bereichen quartärer Ablagerungen auftreten. Eine hohe Priorität erhielten Quellen mit einer Entfernung von unter $150 \mathrm{~m}$ zur Cenoman-Turon-Grenze. Eine Distanz von 150 bis $250 \mathrm{~m}$ entspricht einer mittleren Priorität und Quellen mit einer Distanz darüber hinaus erhielten eine niedrige Priorität.

Im nächsten Schritt wurde in Bereichen, in denen zwei Quellen nah beieinander liegen, die Quelle mit der höheren Priorität ausgewählt. In Fällen, wo zwei Quellen die gleiche Prioritätsstufe aufwiesen, fiel die Wahl auf die Quelle, deren Information laut Quellkataster NRW von der Datenbank Naturschutz des Landesamtes für Naturschutz, Umweltschutz und Verbraucherschutz Nordrhein-Westfallen (DB LANUV) stammt, da eine Vielzahl der ausgewählten Quellen dies als Herkunft aufweisen und somit die Datengrundlage weitestgehend vereinheitlicht werden konnte. 
Abb. 5 Darstellung der Höhenlagen der finalen Auswahl der hydraulischen Tiefpunkte in einer West-Ost-Betrachtung für den Süd- (S) und Ostrand (E) sowie für den Nordrand $(\mathrm{N})$ des Münsterländer Kreidebeckens

Fig. 5 Diagram of the hydraulic pressure points elevation in a west-east view at the south (S), east $(\mathrm{E})$ and north $(\mathrm{N})$ boundary of the Münsterländer Kreidebecken

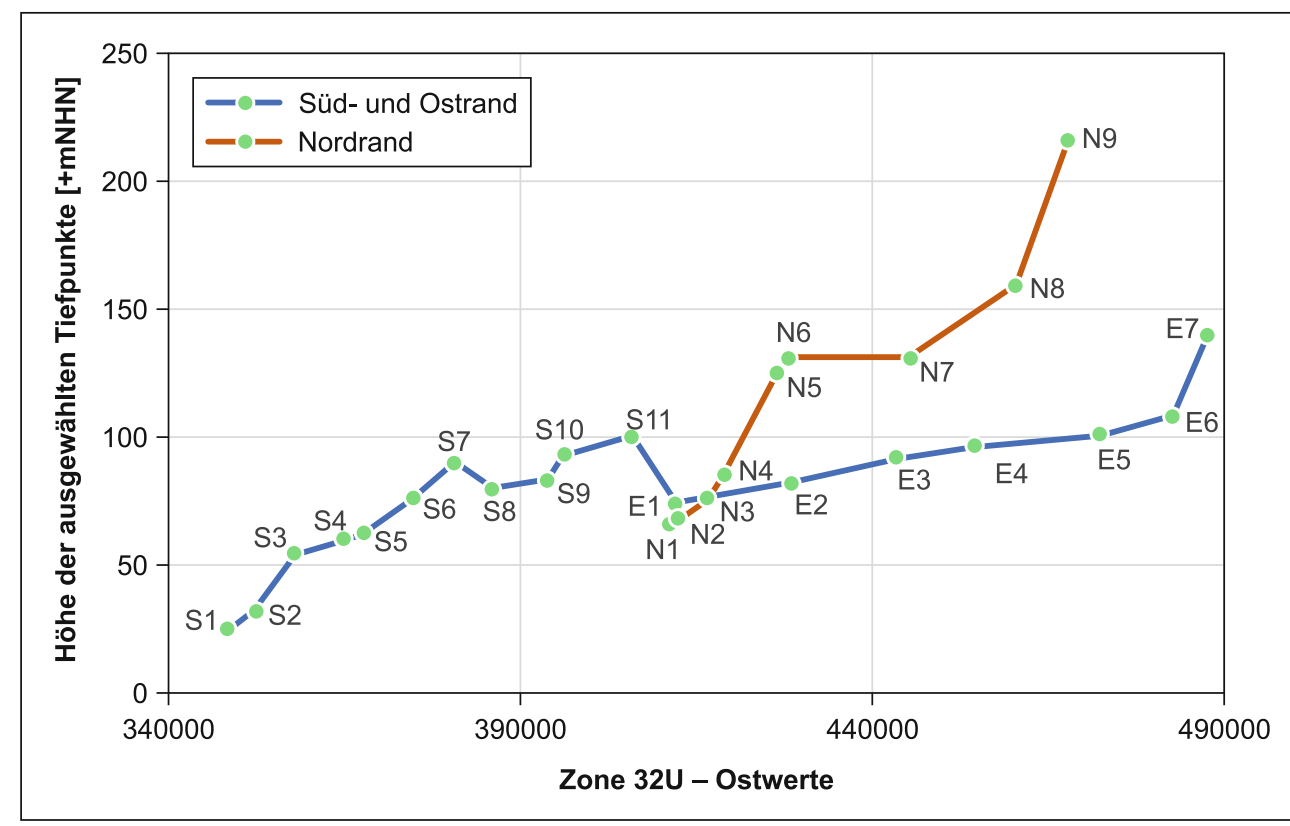

Die Quellen wurden für jedes Blattgebiet (Tecklenburg, Hasbergen, Lengerich, Bockhorst, Halle, Bielefeld, Brackwede) von Westen nach Osten nummeriert und farblich je nach Prioritätsstufe markiert.

Beispielhaft wird das Auswahlverfahren der Quellen am Nordrand für das Blattgebiet Lengerich beschrieben. In Abb. 4 ist die Auswahl von Quellen und deren Priorisierung für das Blattgebiet Lengerich dargestellt. In diesem treten mehrere Quellen südlich entlang der Cenoman-Turon-Grenze auf. 13 Quellen wurden hier für eine nähere Betrachtung markiert. Drei Quellen erhielten eine hohe Priorität und gelangten somit in die nähere Auswahl. Da die Quellen L5 und L6 nah beieinander liegen, wurde die Quelle L6 vom DB LANUV im Quellkataster NRW neben der Quelle L2 in die finale Auswahl der Quellen übernommen.

Am Nordrand liegt zwischen den Blattgebieten Lengerich und Bockhorst ein Einschnitt durch das Bundesland Niedersachsen vor, der dazu führt, dass in diesem Bereich keine vergleichbaren Informationen zu den Quellen aus dem Quellkataster NRW vorliegen. Daher wurde in diesem Gebiet eine Alternative zur Ableitung einer hydraulischen Druckhöhe gewählt. Dabei handelt es sich um Schnittpunkte von zwei Gewässerverläufen, die die Cenoman-TuronGrenze lotrecht schneiden und damit als druckentlastende Punkte des Systems fungieren. Für die beiden Schnittpunkte wurden Höhendaten vom NIBIS ${ }^{\circledR}$ Kartenserver (NIBIS $^{\circledR}$ Kartenserver 2014) abgerufen. Die Auswahl wurde auf einen Schnittpunkt ausgedünnt. Hierbei spielte die Entfernung zu den nächstgelegenen Quellen, die in die finale Auswahl eingehen, sowie die Höhe der Schnittpunkte eine Rolle. Um eine ausgeglichene Verteilung der hydraulischen
Druckpunkte am Nordrand zu erreichen, fiel die Wahl auf den Schnittpunkt N7, welcher sich zudem aufgrund seiner Höhenlage, die der im Westen gelegenen „Brüggelief“Quelle entspricht, in den allgemein ansteigenden Trend vorteilhaft einfügt. Es wurden von 32 priorisierten Quellen neun hydraulische Druckpunkte am Nordrand final ausgewählt und von N1 bis N9 durchnummeriert (siehe Abb. 5 und Tab. 1 im Anhang). Die Höhenniveaus der ausgewählten Druckpunkte steigen hier in einer West-OstBetrachtung von $+66 \mathrm{mNHN}$ auf $+216 \mathrm{mNHN}$ an. Westlich des Einschnitts durch das Bundesland Niedersachsen sind im Gegensatz zum Osten des Untersuchungsgebiets mehr Quellen aufgrund ihrer hohen Priorität in die finale Auswahl eingegangen. Im Verlauf von Westen nach Osten ist ein kontinuierlicher Anstieg in der Höhenlage der Quellen/Gewässer zu verzeichnen. Zwischen den Quellen Menebröcker und der Felsenquelle sowie der Quelle des östl. Klosterbaches und der Fosbergquelle sind stärkere Anstiege von ca. $40-50 \mathrm{~m}$ zu verzeichnen.

\section{Ostrand des Kreidebeckens}

Zur Ableitung von Druckhöhen im nahezu gesamten Münsterländer Kreidebecken wurde auch am Ostrand des Münsterländer Kreidebeckens eine Auswahl an hydraulischen Druckpunkten getroffen. Für die Herleitung der Druckhöhen für den regionalen unteren Grundwasserleiter am Ostrand des Münsterländer Kreidebeckens wurde die Betrachtung der (hydro-)geologischen Situation allgemeiner gehalten, da sich das Gebirge hier im bergbaulich unbeeinflussten, nahezu unverritzten Zustand befindet. Für das Hellweggebiet und die Paderborner Hochfläche wurden 
keine einzelnen Blattgebiete betrachtet, sondern hydrogeologische Karten mit größeren Maßstabszahlen sowie weitere Literaturquellen für ausgewählte Regionen als Datengrundlage herangezogen.

Zur Ableitung einer Druckhöhe im Hellweggebiet kommen zwei geologische Schichtgrenzen in Frage. Hierbei handelt es sich zum einen um die Karbon-Kreide-Grenze, welche als Basis zur Ableitung von Druckhöhen am Südrand des Kreidebeckens diente, und zum anderen um den Übergang zum Emscher-Mergel.

Nach Informationen der HÜK 250 (Hydrogeologische Übersichtskarte von Deutschland 1:250.000) vollzieht sich eine Veränderung der Durchlässigkeiten im Raum Werl für die Turon-Normalfazies (02M 4E; Kluft) im Westen mit einer mäßigen Durchlässigkeit der Durchlässigkeitsklasse $4\left(k_{\mathrm{f}}\right.$-Wert $\left.10^{-5}-10^{-4} \mathrm{~m} / \mathrm{s}\right)$ zu einer mittleren bis mäßigen Durchlässigkeit von $9\left(k_{\mathrm{f}}\right.$-Wert $\left.10^{-5}-10^{-3} \mathrm{~m} / \mathrm{s}\right)$ für die $L a$ marcki- und Labiatus-Schichten (02M 4A; Kluft/Karst) östlich von Werl. Ebenso wechseln die SchloenbachiSchichten von unverkarstet (02M 3C; Kluft) im Westen mit einer mäßigen Durchlässigkeit der Durchlässigkeitsklasse 4 $\left(k_{\mathrm{f}}\right.$-Wert $\left.10^{-5}-10^{-4} \mathrm{~m} / \mathrm{s}\right)$ zu verkarstet (02M 3B; Kluft/Karst) im Osten mit einer mittleren bis mäßigen Durchlässigkeit der Durchlässigkeitsklasse $9\left(k_{\mathrm{f}}\right.$-Wert $\left.10^{-5}-10^{-3} \mathrm{~m} / \mathrm{s}\right)(\mathrm{BGR}$ 2020).

Dieser Wechsel der Durchlässigkeiten in den Schichten sowie die Erkenntnisse aus der bereits weit vorangeschrittenen Flutung des Bergwerks Königsborn in Unna veranlassten zu der Überlegung, bereits im Raum Unna auf die Grenze zum Emscher-Mergel zu wechseln. Hier findet die Entlastung des unteren regionalen Grundwasserleiters über die Quellen in Unna-Mühlhausen statt. Dementsprechend wurde eine Quelle in diesem Gebiet als hydraulischer Tiefpunkt für die Interpolation ausgewählt. Die Auswahl erfolgte anhand der in ArcGIS eingeladenen Informationen des Quellkatasters NRW und fiel auf die Quelle mit der Bezeichnung „Quelle bei Unna-Mühlhausen“ (E1).

Als hydraulische Druckpunkte dienen im weiteren Verlauf des Ostrandes, wie im Raum Unna, eine Auswahl an Quellen der Westfälischen Quellenlinie entlang des Hellweges, die zu einer Druckentlastung des unteren regionalen Grundwasserleiters des Münsterländer Kreidebeckens führen. Zwischen Werl und Erwitte erfolgt eine Auswahl von Quellen in einem gleichmäßigen Abstand mittels Sichtung verschiedener Literaturquellen, wie beispielweise „Quellen in Westfalen" von Göbel und Meßer (2014) und der Hydrogeologischen Karte 1:100.000 von Gütersloh (Geologisches Landesamt Nordrhein-Westfalen 1979), die eine Auflistung von „Quellen am Hellweg“ beinhaltet.

Im Osten des Münsterländer Kreidebeckens schließt die Paderborner Hochfläche, welche aus den Schichten der Oberkreide aufgebaut ist, an die Hellwegbörden an. Als gute Grundwasserleiter im Bereich Paderborn werden die Festgesteine des Cenoman-Pläners, Cenoman-Kalks, der Lamarcki-Schichten, der Scaphiten-Pläner sowie der Schloenbachi-Pläner eingestuft. Als Grundwassernichtleiter fungieren der Labiatus-Mergel und der EmscherMergel. Letztere sind an ihrer Ausstrichgrenze durch das Auftreten größerer Karstquellen markiert (Geologisches Landesamt Nordrhein-Westfalen 1972).

Somit sind hier, wie auch am Nordrand im Blattgebiet Tecklenburg, zwei wichtige hydrogeologische Grenzen am Übergang vom Cenoman zum Turon und vom Turon zum Coniac ausgebildet, welche durch das Auftreten von Quellenlinien markiert werden. Im Folgenden wurden als druckentlastende Punkte des unteren regionalen Grundwasserleiters eine Auswahl an Stauquellen entlang der Grenze TuronConiac zwischen Salzkotten und Bad Lippspringe gewählt, die nach Wisotzky et al. (2018) teilweise große Quellschüttungen aufweisen.

Die Wahl der Quellen an der Turon-Coniac-Grenze knüpft an die Annahme, die ab dem Blattgebiet Unna bis zu den Quellen in Erwitte getroffen wurde, an. Am Übergang zu den Betrachtungen am Nordrand sowie Südrand entsteht damit ein hydrogeologisch nachvollziehbar beschriebener Sprung in der Auswahl der Schichtgrenze zur Ableitung der hydraulischen Druckhöhe (siehe Abb. 5).

Am Ostrand wurden zwischen Unna und Bad-Lippspringe insgesamt sieben Quellen als hydraulische Druckpunkte mit Druckhöhen im Bereich zwischen +74 mNHN und +140 mNHN final ausgewählt und von E1 bis E7 durchnummeriert (siehe Abb. 5 und Tab. 1 im Anhang).

Die Ost- und Nordwerte wurden mittels ,Add XY Coordinates" in ArcGIS ermittelt. Die Höhenangaben der hydraulischen Druckpunkte entstammen dem Digitalen Geländemodell DGM1 (Landesvermessung NRW 2020).

\section{Interpolation der hydraulischen Druckfläche}

Auf Grundlage der gewählten hydraulischen Druckpunkte am Süd-, Nord- und Ostrand des Beckens (siehe Tab. 1 im Anhang) erfolgte in ArcGIS eine räumliche Interpolation einer hydraulischen Druckfläche in Form einer RasterOberfläche, die nahezu das gesamte Innere des Münsterländer Kreidebeckens abdeckt.

Die Interpolation der Druckfläche erfolgte mittels verschiedener Interpolationsverfahren. Als Eingangsdaten fungierten bei allen Verfahren die Tiefpunkte am Südrand und die Quellen (bzw. Gewässer) am Nord- und Ostrand. Die finale Auswahl fiel auf das Ergebnis des Interpolationsverfahrens „Natural Neighbor“. Bei der Interpolationsmethode „Natural Neighbor“ ergibt sich der Wert eines neuen Punktes, indem die Flächeninhalte der Überschneidungen mit den Nachbarzellen im Verhältnis zum Gesamtflächeninhalt der neuen Zelle als Gewichtungsfaktoren der Inter- 


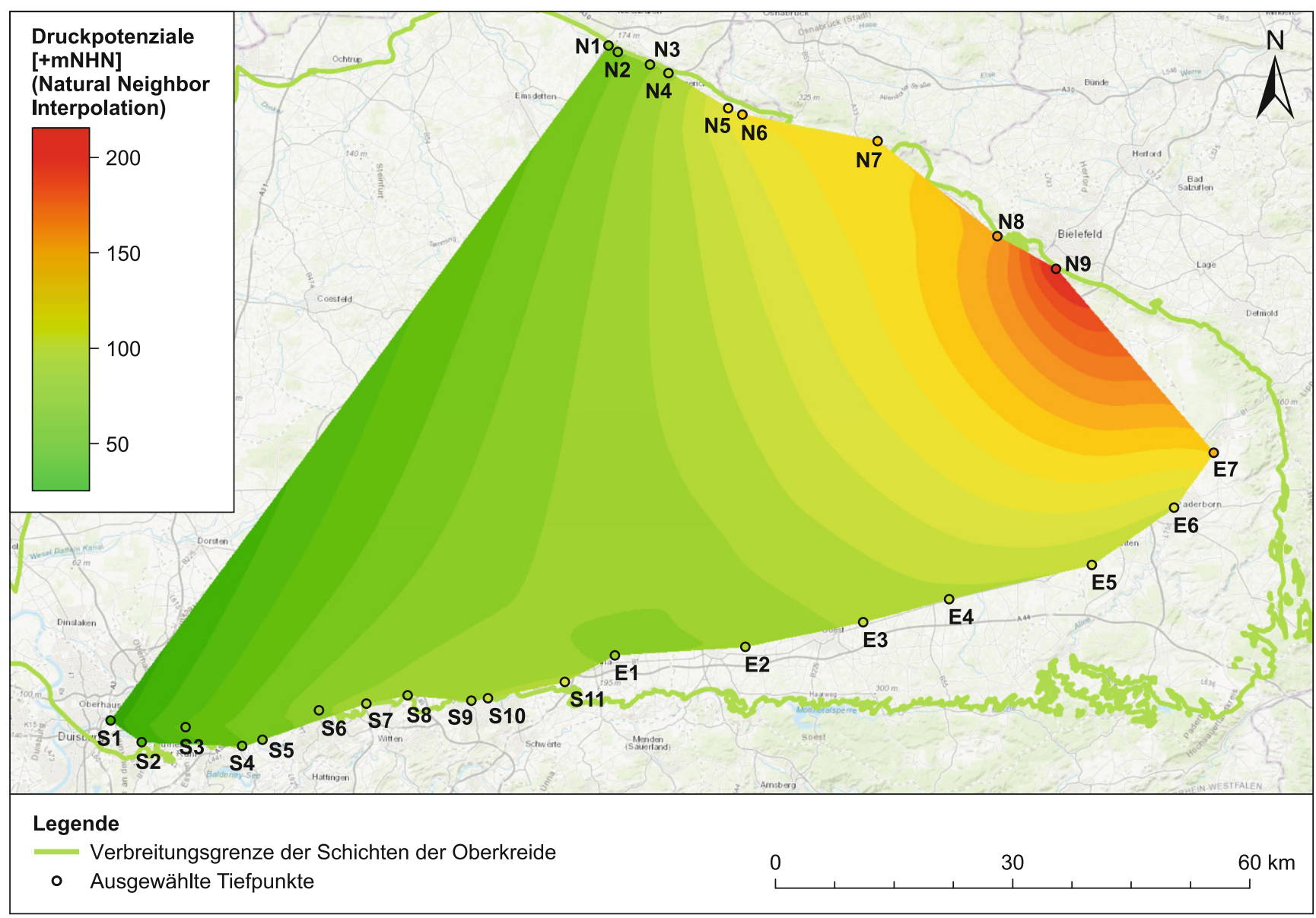

Abb. 6 Darstellung des Interpolationsergebnisses des Verfahrens „Natural Neighbor“ (unter Verwendung der Daten von esri (2020); Geowissenschaftliche Daten: Geologisches 3D-Landesmodell NRW 2012 (Geologischer Dienst NRW 2019); LANUV (2020))

Fig. 6 Representation of the interpolation result of the natural neighbor method (using data from esri (2020); geoscientific data: Geologisches 3DLandesmodell NRW 2012 (Geologischer Dienst NRW 2019); LANUV (2020))

polation verwendet werden. Für die Interpolation mit dem Verfahren „Natural Neighbor“ wurde für die „Output cell size“ eine Kantenlänge von $100 \mathrm{~m}$ gewählt. Die interpolierte Raster-Oberfläche weist eine relativ gleichmäßige Verteilung und Abstufung der Isolinien und Druckpotenziale auf (siehe Abb. 6). Die Fläche verläuft fast überall durch die Eingabepunkte.

Die interpolierte Druckpotenzialfläche bildet die hydrostatische Situation, welche sich aus den Randdruckhöhen ergibt, im unteren regionalen Grundwasserleiter des Münsterländer Kreidebeckens ab. Die Fläche weist ein generelles Gefälle nach Westen mit ihrem höchstgelegenen Druckpunkt im Nordosten (N 9 Fosbergquelle bei Bielefeld) und ihrem niedrigsten Druckpunkt im Südwesten auf.

\section{Diskussion}

Die interpolierte Druckpotenzialfläche hängt von der Wahl der hydraulischen Druckpunkte am Rand des Münsterländer
Kreidebeckens ab. Für alle drei betrachteten Ränder (Nord, Süd und Ost) des Münsterländer Kreidebeckens wurde die (hydro-)geologischen Situation betrachtet und Annahmen für potenzielle hydraulische Druckpunkte im Falle eines ungehinderten Grubenwasseranstiegs getroffen.

Am Nord- und Südrand wurden unterschiedliche hydrogeologische Grenzen zur Ableitung der druckentlastenden Tiefpunkte verwendet. Am Südrand wurden Tiefpunkte entlang der Grenze zwischen Karbon und Oberkreide gewählt. Am Nordrand wurden die Druckhöhen von Quellen abgeleitet, die innerhalb der Schichten der Oberkreide, entlang des Übergangs vom Cenoman zum Turon, liegen. Die hydrogeologische Grenze am (Süd-)Ostrand bildet das Verbindungsglied zwischen dem Nord- und Südrand. Hier wurde die Grenze zum Emscher-Mergel als Orientierung zur Auswahl von Quellen, die als Tiefpunkte der Druckentlastung im unteren Grundwasserleiter dienen, gewählt. Vor dem Hintergrund der Betrachtung eines ,worst-case“-Szenarios zum Grubenwasseranstieg wurde - mit Ausnahme am Ostrand - die stratigraphisch höher liegende, vom Becken- 
inneren weiter entfernt liegende Grenze gewählt. Bei der Wahl der Tiefpunkte zur Druckentlastung des aufsteigenden Grubenwassers im Karbon, am Übergang zu dem überwiegend als Grundwassernichtleiter fungierenden Essener Grünsand der Oberkreide, wurde darauf geachtet, dass keine Über- oder Unterschätzung der Druckhöhen im ,worstcase"-Szenario erfolgte.

Am Südrand wurde, durch den Einbezug von Stollenmundlöchern als Tiefpunkte, zudem der Einfluss ehemaliger Entwässerungselemente, die durch den Bergbau im Steinkohlenrevier der Ruhr entstanden sind und für die die Annahme getroffen wurde, dass diese auch heute noch hydraulisch wirksam sind, berücksichtigt. Damit wird im ,worstcase"-Szenario nicht der historische Zustand im Vorfeld der bergbaulichen Beeinflussung, sondern ein bereits anthropogen beeinflusster und unumkehrbarer Zustand abgebildet. Das durch bergbauliche Einflüsse geschaffene subregionale hydrologische System beeinflusst den im gesamten Münsterländer Kreidebecken verbreiteten unteren Grundwasserleiter der Kreide. Dies wird durch die Verbindung der hydraulischen Druckpunkte am Nord- und Südrand sowie Ostrand im Zuge der Interpolation verdeutlicht.

Die Wahl der Quellen an der Grenze vom Cenoman-Kalk zu den labiatus-Schichten am Nordrand des Münsterländer Kreidebeckens unterliegt gewissen Unsicherheiten in Bezug auf die exakte Zuordnung der Quellen zum Grundwasserleiter des Cenoman-Kalks. Die ausstreichenden Schichten der Oberkreide am Nordrand haben eine geringe Ausstrichbreite, sodass die Grundwasserstockwerke und die dazugehörigen Quellen eng beieinander liegen und der Fehler gering wäre. Am (Süd-)Ostrand des Münsterländer Kreidebeckens wurde die Betrachtung der (hydro-)geologischen Situation allgemeiner gehalten. Aufgrund der mangelnden Datengrundlage wurde eine allgemeine Annahme für das Hellweggebiet und die Paderborner Hochfläche getroffen. Detailliertere Daten - insbesondere im Verbreitungsgebiet der Senne-Sande - würden hier eine höhere Detailschärfe ermöglichen.

Im Westen des Münsterländer Kreidebeckens tritt der untere Grundwasserleiter zwischen Borken und Mülheim aufgrund der Überdeckung durch tonige tertiäre Schichten nicht zutage (Struckmeier 1990). Für den Westrand des Beckens liegen keine plausiblen Punkte vor, an denen Grundwasser aufquellen könnte und zu einer Druckentlastung führen würden. Demnach entfiel die Auswahl von hydraulischen Druckpunkten des unteren regionalen Grundwasserleiters in dieser Region.

Die Auswahl der hydraulischen Druckpunkte am Nord-, Süd- und Ostrand erfolgte mit relativ gleichmäßigem Abstand zueinander. Am Südrand betragen die Abstände 2 bis $10 \mathrm{~km}$, am Süd-Ostrand 8,5 bis $18,5 \mathrm{~km}$, am Nord-Ostrand bis zu $19 \mathrm{~km}$ und im Nordwesten 1,5 bis $8,7 \mathrm{~km}$. Im Verbreitungsgebiet der Senne-Sande nimmt der Abstand den größ- ten Wert mit 30,6 km ein. Hier tritt auch ein großer hydraulische Druckunterschied von $76 \mathrm{~m}$ zwischen der Fosbergquelle (N9) in Bielefeld-Brackwede und der Lippe-Quelle (E7) in Bad Lippspringe auf. Dies ist dadurch begründet, dass im Verbreitungsgebiet der Senne-Sande die Ableitung hydraulischer Druckpunkte erschwert ist.

Die höchst gelegenen hydraulischen Druckpunkte der gesamten Auswahl befinden sich im Nordosten. Im Südwesten treten die niedrigsten Druckhöhen auf. Die interpolierte Druckpotenzialfläche im Münsterländer Kreidebecken wird maßgeblich von diesen Punkten beeinflusst. Das Ergebnis unterstützt aber auch die Annahme, dass der untere Grundwasserleiter von Norden her gespeist wird und eine Druckentlastung des unteren regionalen Grundwasserleiters nach Südwesten, im Bereich des sandigen Emscher-Mergels, bevor dieser von undurchlässigeren tertiären Schichten überdeckt wird, gerichtet ist.

Die Fläche wird durch die hydraulische Durchlässigkeit im Karbon deformiert, diese spielt im Interpolationsalgorithmus aber keine Rolle. Daher ist die Annahme eines homogenen Karbons wohl die gravierendste Ungenauigkeit.

\section{Fazit}

Die hydraulischen Druckpunkte, die im Hinblick auf den Fall eines ungehinderten Grubenwasseranstiegs am Süd-, Nord- und Ostrand ausgewählt wurden und die daraus resultierende hydraulische Druckfläche, stellen „natürliche“ unregulierte Druckverhältnisse im unteren regionalen Grundwasserleiter des Münsterländer Kreidebeckens dar, welche jedoch unter anderem durch den Einbezug der Erbstollen passiv durch die langjährigen bergbaulichen Tätigkeiten im Bereich des Steinkohlenabbaugebietes an der Ruhr beeinflusst werden. Diese auch im Falle eines ungehinderten Grubenwasseranstiegs anhaltende anthropogene Beeinflussung durch die im Zuge der bergbaulichen Tätigkeiten angelegten Entwässerungsstrukturen, trägt somit zu der Ausbildung subregionaler hydraulischer Verhältnisse am Südrand des Münsterländer Kreidebeckens bei.

Allgemein befinden sich am Nord- und Südrand die höchstgelegenen hydraulischen Druckpunkte im Osten und die niedrigsten Druckpunkte im Westen des jeweiligen Randes. Die Druckhöhen weisen somit eine Abnahme von Osten nach Westen auf. Dementsprechend kann ein nach Westen gerichtetes hydraulisches Druckgefälle angenommen werden. Der höchste hydraulische Druckpunkt der finalen Auswahl für die Betrachtung zwischen dem Nord- und Südrand, auch unter Hinzunahme der Quellen am Ostrand, liegt im Nordosten des Beckens (Fosbergquelle bei Bielefeld). Der niedrigste Druckpunkt befindet sich hingegen auf der gegenüberliegenden Beckenseite im Südwesten (Ruhrbogen in Oberhausen). Aufgrund der La- 
ge dieser Hoch- und Tiefpunkte des Beckens kann eine gewisse Fokussierung der Druckentlastung im Südwesten bzw. Westen angenommen werden, welche sich in der mittels ArcGIS interpolierten Raster-Oberfläche widerspiegelt. Aufgrund des mächtigen Emscher-Mergels handelt es sich bei der interpolierten Druckpotenzialfläche um gespannte Verhältnisse im unteren Grundwasserleiter, die keineswegs zu einer Überflutung des Münsterländer Kreidebeckens führen.
Die Druckpotenzialfläche ist zukünftig für die ,worstcase"-Betrachtung von Grubenwasseranstiegsszenarien in stillgelegten Bergwerken oder von aufsteigenden Tiefengrundwässern in oberflächennahe Grundwasserleiter verwendbar.

\section{Anhang}

Tab. 1 Auswahl der hydraulischen Druckpunkte am Süd-, Nord- und Ostrand des Münsterländer Kreidebeckens Table 1 Selection of the hydraulic pressure points at the south, north and east boundary of the Münsterländer Kreidebecken

\begin{tabular}{|c|c|c|c|c|}
\hline$\overline{\mathrm{Bez}}$ & Kriterium & $\begin{array}{l}\text { Höhe } \\
{[+\mathrm{mNHN}]}\end{array}$ & $\begin{array}{l}\text { UTM-Zone } 32 \mathrm{U} \\
\text { Ostwert }\end{array}$ & $\begin{array}{l}\text { UTM-Zone } 32 \mathrm{U} \\
\text { Nordwert }\end{array}$ \\
\hline$\overline{\mathrm{S} 1}$ & 2 (Ruhrbogen in Oberhausen) & 25 & 348272 & 5702915 \\
\hline S2 & 1 (Stollenmundloch „Broicher Erbstollen“) & 32 & 352207 & 5700150 \\
\hline S3 & 3 (Schnittpunkt Borbecker Mühlenbach/Karbon-Kreide-Grenze) & 54 & 357759 & 5702059 \\
\hline S4 & 1 (Stollenmundloch „,Kunstwerk“) & 60 & 364904 & 5699677 \\
\hline S5 & 1 (Stollenmundloch „Mecklingsbänker Erbstollen“) & 62 & 367470 & 5700458 \\
\hline S6 & 3 (Schnittpunkt Marbach/Karbon-Kreide-Grenze) & 76 & 374632 & 5704196 \\
\hline S7 & 1 (Stollenmundloch „Victoriastollen“) & 90 & 380601 & 5705042 \\
\hline S8 & 3 (Schnittpunkt Roßbach/Karbon-Kreide-Grenze) & 80 & 385838 & 5706075 \\
\hline S9 & 2 (Schnittpunkt Emscher/Schondelle) & 83 & 393874 & 5705425 \\
\hline S10 & 2 (Schnittpunkt Emscher/Hörder Bach) & 93 & 395983 & 5705713 \\
\hline $\mathrm{S} 11$ & 1 (Stollenmundloch „Caroliner Erbstollen“) & 100 & 405720 & 5707796 \\
\hline E1 & Quelle bei Unna-Mühlhausen & 74 & 412059 & 5711147 \\
\hline E2 & Quellteich „Der Börn“ & 82 & 428520 & 5712226 \\
\hline E3 & „Ahse-Quelle“ & 92 & 443448 & 5715333 \\
\hline E4 & „Erwitter Spring“ & 96 & 454289 & 5718247 \\
\hline E5 & „Ursprunge 1 Heder-Gruppe $1 “$ & 101 & 472367 & 5722595 \\
\hline E6 & „Warme Paderquelle“ & 108 & 482732 & 5729821 \\
\hline E7 & „Lippe-Quelle“ & 140 & 487761 & 5736769 \\
\hline N9 & Fosbergquelle & 216 & 467809 & 5760045 \\
\hline $\mathrm{N} 8$ & Quelle des östl. Klosterbaches & 159 & 460392 & 5764154 \\
\hline N7 & Schnittpunkt Dissener Bach/Cenoman-Turon-Grenze & 131 & 445253 & 5776187 \\
\hline N6 & „Brüggelief-Quelle“ & 131 & 428170 & 5779548 \\
\hline N5 & „Felsenquelle“ & 125 & 426364 & 5780352 \\
\hline N4 & Menebröcker & 85 & 418805 & 5784798 \\
\hline N3 & Stapenhorst-Teich & 76 & 416472 & 5785872 \\
\hline $\mathrm{N} 2$ & Am Klee & 68 & 412431 & 5787459 \\
\hline N1 & Ehemaliges Kalksteinbruchgelände „Am Klee“ & 66 & 411232 & 5788282 \\
\hline
\end{tabular}

Quellen: Für alle genutzt: Digitales Geländemodell DGM1 (Landesvermessung NRW 2020); S3, S6, S8: Oberkreidegrenze-shape (Geologischer Dienst Nordrhein-Westfalen 2019a); 2, S4, S5, S7, S11: Stollenmundlöcher (Tiedt 2020); S1, S3, S6, S8, S9, S10: Gewässerstationierungskarte (LANUV 2020); E1-7: Quellkataster NRW (Geologischer Dienst Nordrhein-Westfalen 2019b); N1-N6, N8, N9: Quellkataster NRW (Geologischer Dienst Nordrhein-Westfalen 2019b); N1-N4: Tecklenburg (Geologisches Landesamt Nordrhein-Westfalen 1970); N5, N6: Lengerich (Preussische geologische Landesanstalt 1935); N8: Halle (Königlich Preussische Geologische Landesanstalt 1926); N9: Brackwede (Geologisches Landesamt Nordrhein-Westfalen 1982); N7: Gewässer Niedersachsen (NIBIS ${ }^{\circledR}$ Kartenserver 2014); N7: Gewässerstationierungskarte (LANUV 2020) 
Danksagung Diese Arbeit basiert auf Ergebnissen des Forschungsprojektes „Auswirkungen eines ungehinderten Grubenwasseranstiegs sowie der Einstellung aller Poldermaßnahmen im Einzugsgebiet von Emscher und Lippe auf das oberflächennahe Grundwasser und die oberirdischen Gewässer". Wir bedanken uns für die Förderung durch das Deutsche Stiftungszentrum GmbH im Stifterverband „Forum Bergbau und Wasser“ (Förderkennzeichen T0518/33420/2019) und dem zuständigen Kurator Prof. Dr. Christian Wolkersdorfer.

Barbara Fister und Patrick Cantauw danken wir für die Erstellung und Überarbeitung der Abbildungen.

Funding Open Access funding enabled and organized by Projekt DEAL.

Open Access Dieser Artikel wird unter der Creative Commons Namensnennung 4.0 International Lizenz veröffentlicht, welche die Nutzung, Vervielfältigung, Bearbeitung, Verbreitung und Wiedergabe in jeglichem Medium und Format erlaubt, sofern Sie den/die ursprünglichen Autor(en) und die Quelle ordnungsgemäß nennen, einen Link zur Creative Commons Lizenz beifügen und angeben, ob Änderungen vorgenommen wurden.

Die in diesem Artikel enthaltenen Bilder und sonstiges Drittmaterial unterliegen ebenfalls der genannten Creative Commons Lizenz, sofern sich aus der Abbildungslegende nichts anderes ergibt. Sofern das betreffende Material nicht unter der genannten Creative Commons Lizenz steht und die betreffende Handlung nicht nach gesetzlichen Vorschriften erlaubt ist, ist für die oben aufgeführten Weiterverwendungen des Materials die Einwilligung des jeweiligen Rechteinhabers einzuholen.

Weitere Details zur Lizenz entnehmen Sie bitte der Lizenzinformation auf http://creativecommons.org/licenses/by/4.0/deed.de.

\section{Literatur}

BGR: Geoviewer. Hydrogeologische Übersichtskarte HUEK250 (2020). https://geoviewer.bgr.de/mapapps4/resources/apps/ geoviewer/index.html?lang=de \&tab=grundwasser \& cover $=$ grundwasserDE, Zugegriffen: 3. Juni 2020

Coldewey, W.G., Wesche, D.: Hydrogeologische und gesteinsphysikalische Eigenschaften der Emscher-Formation im Hinblick auf den Steinkohlenbergbau des Ruhrgebietes. Z. Grundwasser 22(3), 175-183 (2017)

esri: World Topographic Map (2020). https://services.arcgisonline. com/ArcGIS/rest/services/World_Topo_Map/MapServer, Zugegriffen: 2. Juli 2020

Geologischer Dienst Nordrhein-Westfalen: Hydrogeologischen Karte von Nordrhein-Westfalen, Blatt C 3712 Ibbenbüren. Geologischer Dienst Nordrhein-Westfalen, Krefeld (2001)

Geologischer Dienst Nordrhein-Westfalen: Geowissenschaftliche Daten. Geologisches 3D-Landesmodell NRW 2018 (2021). https:// www.govdata.de/dl-de/by-2-0. Zugegriffen: 17. Okt. 2021

Geologischer Dienst Nordrhein-Westfalen: Quellkataster NRW auf Basis der Gewässerstationierungskarte (2019b). https://www. opengeodata.nrw.de/produkte/geologie/geologie/HK/ISQuellen/, Zugegriffen: 17. Okt. 2019

Geologischer Dienst Nordrhein-Westfalen: Verbreitung der Unterkreide, Stand 2012 aus Landesmodell NRW, Stand 2019 (2019a). https://www.govdata.de/dl-de/by-2-0. Zugegriffen: 17. Okt. 2021

Geologisches Landesamt Nordrhein-Westfalen: Geologische Karte von Nordrhein-Westfalen. GK 3712 Tecklenburg mit Erläuterungen und Hydrogeologischer Karte. Geologisches Landesamt Nordrhein-Westfalen, Krefeld (1970)

Geologisches Landesamt Nordrhein-Westfalen: Hydrogeologischen Karte des Kreises Paderborn und der angrenzenden Gebiete L 4318 mit Erläuterungen. Geologisches Landesamt NordrheinWestfalen, Krefeld (1972)
Geologisches Landesamt Nordrhein-Westfalen: Hydrogeologische Karte von Nordrhein-Westfalen, Blatt C 4314 Gütersloh mit Erläuterungen, 1. Aufl. Geologisches Landesamt NordrheinWestfalen, Krefeld (1979)

Geologisches Landesamt Nordrhein-Westfalen: Geologische Karte von Nordrhein-Westfalen. GK 4017 Brackwede mit Erläuterungen. Geologisches Landesamt Nordrhein-Westfalen, Krefeld (1982)

Geologisches Landesamt Nordrhein-Westfalen: Geologische Karte von Nordrhein-Westfalen. GK 4507 Mülheim an der Ruhr mit Erläuterungen und Hydrogeologischer Karte. Geologisches Landesamt Nordrhein-Westfalen, Krefeld (1986)

Geologisches Landesamt Nordrhein-Westfalen: Geologische Karte von Nordrhein-Westfalen. GK 4510 Dortmund mit Erläuterungen und Hydrogeologischer Karte. Geologisches Landesamt NordrheinWestfalen, Krefeld (1987)

Geologisches Landesamt Nordrhein-Westfalen: Geologische Karte von Nordrhein-Westfalen. GK 4509 Bochum mit Erläuterungen, geologischen Schnitten und Hydrogeologischer Karte. Geologisches Landesamt Nordrhein-Westfalen, Krefeld (1988)

Geologisches Landesamt Nordrhein-Westfalen: Ingenieurgeologische Karte. IK 4508 Essen mit Erläuterungen. Geologisches Landesamt Nordrhein-Westfalen, Krefeld (1992)

Göbel, P., Meßer, J.: Quellen in Westfalen. Geographisch-landeskundlicher Atlas von Westfalen Landesnatur. Aschendorff, Münster (2014)

Königlich Preußische Geologische Landesanstalt: Erläuterungen zur Geologischen Karte von Preußen und benachbarten deutschen Ländern. GK 3916. Königlich Preußische Geologische Landesanstalt, Halle i. W.. Berlin (1926)

Landesamt für Bergbau, Energie und Geologie. Hannover: NIBIS ${ }^{\circledR}$ Kartenserver: Topografien Niedersachsen (LGLN) (2014). https:// nibis.lbeg.de/cardomap3/, Zugegriffen: 8. März 2020

Landesvermessung NRW: Digitales Geländemodell DGM1 (2020). https://www.opengeodata.nrw.de/produkte/geobasis/hm/dgm1_ xyz/, Zugegriffen: 15. Okt. 2019

LANUV: Gewässerstationierungskarte des Landes Nordrhein-Westfalen. WMS-Dienst (2020). https://www.lanuv.nrw.de/umwelt/ wasser/oberflaechengewaesserfluesse-und-seen/fliessgewaesser/ gewaesser-stationierungskarte, Zugegriffen: 3. Juli 2020

Meßer, J.: Auswirkungen der Urbanisierung auf die GrundwasserNeubildung im Ruhrgebiet unter besonderer Berücksichtigung der Castroper Hochfläche und des Stadtgebietes Herne. Berichte aus Forschung und Entwicklung, Bd. 58. DMT, Bochum (1997)

Michel, G.: Grundwasser - Dargebot, Nutzung und Gefährdung. In: Geologie im Münsterland, S. 118-127. Geologisches Landesamt Nordrhein-Westfalen, Krefeld (1995)

Müller, W.: Das Deckgebirge. In: Hahne, C., Schmidt, R. (Hrsg.) Die Geologie des niederrheinisch-westfälischen Steinkohlengebietes, S. 72-98. Glückauf, Essen (1982)

Preußische geologische Landesanstalt: Geologische Karte von PreuBen und benachbarten deutschen Ländern. Erläuterungen zu Blatt 3813. Lengerich, Berlin (1935)

Struckmeier, W.: Wasserhaushalt und hydrologische Systemanalyse des Münsterländer Beckens. Schriftenreihe des Landesamtes für Wasser und Abfall NRW, Bd. 45. LWA, Düsseldorf (1990)

Tiedt, M.: Der frühe Bergbau an der Ruhr. Spurenkarten (2012). http:// www.ruhrkohlenrevier.de/karten.html, Zugegriffen: 2. März 2020

Tiedt, M.: Stollenmundlöcher. Ost- und Nordwerte, Bezeichnungen der Erbstollen und Jahreszahl der Fertigstellung. Unveröffentlicht (2020)

Wisotzky, F., Cremer, N., Lenk, S.: Angewandte Grundwasserchemie, Hydrogeologie und hydrogeochemische Modellierung. Grundlagen, Anwendungen und Problemlösungen. Springer, Berlin, Heidelberg (2018)

Hinweis des Verlags Der Verlag bleibt in Hinblick auf geografische Zuordnungen und Gebietsbezeichnungen in veröffentlichten Karten und Institutsadressen neutral. 